\title{
Principles and Applications of Energy-Filtered Scanning CBED for Ferroelectric Domain Imaging and Symmetry Determination
}

\author{
Yu-Tsun Shao ${ }^{1}$, Kyou-Hyun $\mathrm{Kim}^{2}$ and Jian-Min Zuo ${ }^{1,3}$ \\ 1. Department of Materials Science and Engineering, University of Illinois, Urbana, Illinois 61801, USA \\ 2. Advanced Process and Materials R\&BD Group, Korea Institute of Industrial Technology, Incheon, \\ 406-840, Korea \\ 3. Frederick Seitz Materials Research Laboratory, University of Illinois, Urbana, Illinois 61801, USA
}

The presence of nanodomains is considered to play an important role in determining the optical properties, dielectric permittivity and polarization switching in relaxor-based ferroelectric crystals. Thus, understanding the complex nanodomain structure could provide important clues about the mechanisms that produce the extraordinary piezoelectric responses in relaxor-based ferroelectric crystals. However, techniques such as X-ray diffraction (XRD), neutron diffraction and optical microscopy only provide information about the macroscopic or average symmetry by using probe sizes that are much larger than the size of nanodomains. Determining the polarizations of nanodomains are in general performed using piezoresponse force microscopy (PFM). Atomic resolution scanning TEM (STEM) is also used to determine polarization directions in ferroelectric thin films. For the complex nanodomain structure in relaxor-based ferroelectric single crystals, convergent beam electron diffraction (CBED) has been considered as a powerful technique by using a finely focused probe of a few nanometers in diameter or less [1]. Here we use a recently-developed technique called scanning CBED which has the capability of quantifying as well as mapping the local symmetry variations at nanometer scale [2]. This is based on automated recording of the CBED patterns on the CCD camera while scanning over the region of interest with nanometer-sized electron beam and correlation analysis of recorded patterns.

The relaxor-based ferroelectric single crystals of $(1-\mathrm{x}) \mathrm{Pb}\left(\mathrm{Zn}_{1 / 3} \mathrm{Nb}_{2 / 3}\right) \mathrm{O}_{3}-\mathrm{xPbTiO}_{3}(\mathrm{PZN}-\mathrm{xPT})$ are studied here. This system together with PMN-xPT (M for $\mathrm{Mg}$ ) have attracted much research interest due to their superior piezoelectric properties $\left(\mathrm{d}_{33}>2,500 \mathrm{pm} / \mathrm{V}\right.$ and $\left.\mathrm{k}_{33}>0.9\right)$ in the morphotropic phase boundary (MPB) region compared to commercial piezoelectric ceramics [3]. The lead-based relaxor-based ferroelectric crystals are known to have three types of macroscopic symmetry of $\mathrm{R}$ (rhombohedral), $\mathrm{M}$ (monoclinic), and T (tetragonal) at room temperature dependent on composition. The local symmetry in MPB region of relaxor-based ferroelectric crystals, however, is still under debate.

In our study, the scanning CBED was performed at JEOL 2010F TEM, the electron probe had a diameter of about $2.3 \mathrm{~nm}$ in FWHM (full-width at half-maximum). The scanning CBED was performed using a step size of $2 \mathrm{~nm}$, and we recorded $15 \times 15$ data points in a local area of the size $28 \mathrm{~nm} \times 28 \mathrm{~nm}$ inside the specimen. Each CBED patterns were energy-filtered using the post-column Gatan Imaging Filter (GIF) with $10 \mathrm{eV}$ energy window to reduce inelastic scattering effects. Figure 1(a) shows the bright field image of the PZN-8\%PT sample, the yellow square indicates the scanned region. To analyze the patterns, we calculated the correlation coefficients among different CBED patterns in each scanned dataset. To quantify the similarity between two images, we used the normalized cross-correlation coefficient and profile R-factor, $\gamma$ and $\mathrm{R}_{\mathrm{p}}$, respectively, by pixel-to-pixel comparison. We then set the threshold of $\gamma$ manually to obtain a stack of templates from the original scanned image stack. By correlating the generated templates with the original scanned images we thus have the correlation map. Figure 1(b) is the correlation map of scanning CBED patterns, which maps out various polarization 
nanodomains. Figure 2(a) is the representative CBED patterns of the PZN-8\%PT single crystal, the numbers correspond to Fig. 1(b). Figure 2(b) is the simulated CBED pattern on (001) zone axis based on the Bloch-wave method. Thus, scanning CBED combined with correlation analysis and Bloch wave simulations provides a new method for imaging nanodomains in ferroelectric crystals at about $2 \mathrm{~nm}$ resolution.

\section{References:}

[1] K. Kim, D. A. Payne, and J. M. Zuo, Phys. Rev. B 86 (2012), 184113.

[2] K. Kim and J. M. Zuo, Ultramicroscopy 124 (2013), 71.

[3] S. E. Park and T. R. Shrout, J. Appl. Phys. 82 (1997), 1804.

[4] This work is supported by U.S. Department of Energy, Office of Basic Energy Sciences under contract DEFG02-01ER45923.
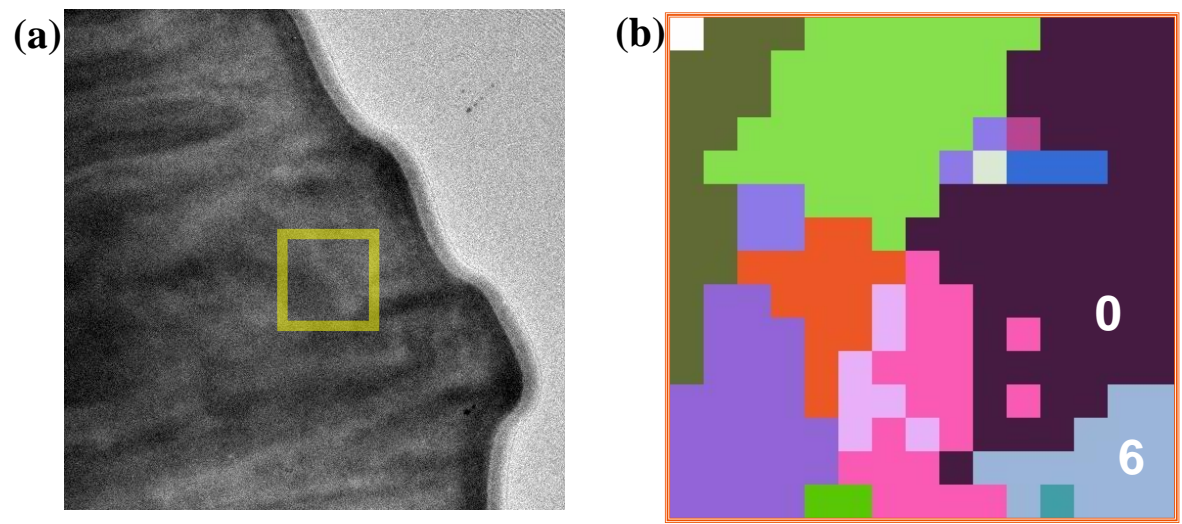

Figure 1. (a) Bright-field image of a 55nm PZN-8\%PT sample. The yellow square represents the region of scanning (b) correlation map of scanning CBED patterns.

(a)

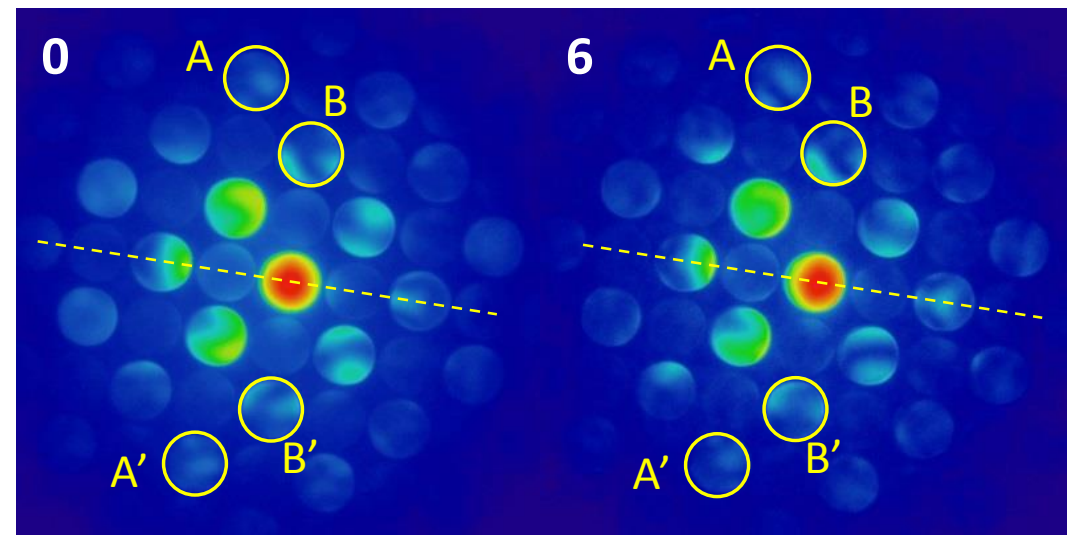

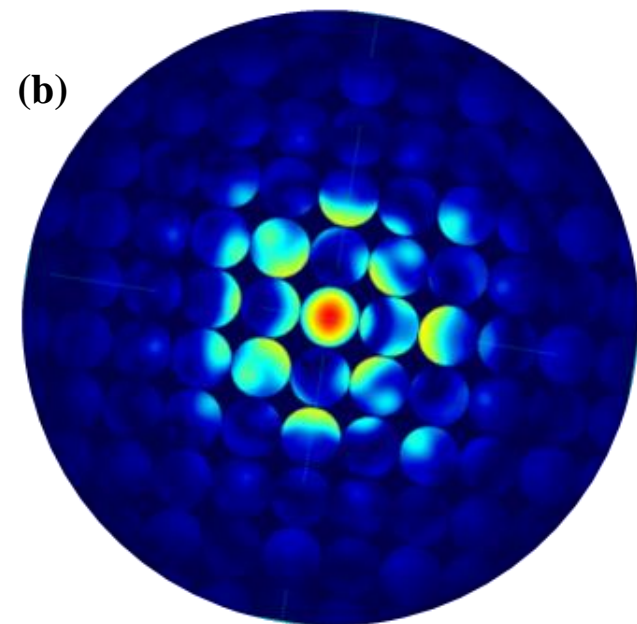

Figure 2. (a) Representative energy-filtered CBED patterns of PZN-8\%PT single crystal, the numbers correspond to the numbers in Fig. 1(b). To quantify the mirror symmetry, we compared two pairs of diffraction disks within each pattern, A-A' and B-B', by calculating the $\gamma$ and Rp of the mirror-applied A (and B) with $A^{\prime}$ (and B') using methods described in ref. [2]. The highest values of $\left(\gamma, R_{p}\right)$ we calculated from $A-A^{\prime}$ of $0^{\text {th }}$ - and $6^{\text {th }}$-template are $(0.95,0.08)$ and $(0.65,0.29)$, respectively. For B-B', the values are $(0.90,0.22)$ and $(0.87,0.34)$. (b) Simulated CBED pattern using the Bloch-wave method. 